\title{
Patterns of Palliative Care Consultation Among Elderly Patients With Cancer
}

\author{
Eric J. Roeland, MD; ; Daniel P. Triplett, $\mathrm{MPH}^{\mathrm{b}}$; Rayna K. Matsuno, PhD, MPH'; Isabel J. Boero, $\mathrm{BS}^{\mathrm{b}}$; \\ Lindsay Hwang, BS ${ }^{b}$ Heidi N. Yeung, MDa; Loren Mell, MD' ; and James D. Murphy, MD, MS
}

\begin{abstract}
Background: The role of palliative care has expanded over the past several decades, although the oncology-specific regional evolution of this specialty has not been characterized at the population-based level. Methods: This study defined the patterns of palliative care delivery using a retrospective cohort of patients with advanced cancer within the SEER-Medicare linked database. We identified 83,022 patients with metastatic breast, prostate, lung, and colorectal cancers. We studied trends between 2000 through 2009, and determined patientlevel and regional-level predictors of palliative care delivery. Results: Palliative care consultation rates increased from $3.0 \%$ in 2000 to $12.9 \%$ in 2009 , with most consultations occurring in the last 4 weeks of life $(77 \%)$ in the inpatient hospital setting. The rates of palliative care delivery were highest in the West (7.6\%) and lowest in the South (3.2\%). The likelihood of palliative care consultation increased with decreasing numbers of regional acute care hospital beds per capita. The use of palliative care consultation increased with increasing numbers of regional physicians. The use of palliative care decreased with increasing regional Medicare expenditure with a $\$ 1,387$ difference per beneficiary between the first and fourth quartiles of palliative care use. Conclusions: Geographic location influences a patient's options for palliative care in the United States. Although the overall rates of palliative care are increasing, future effort should focus on improving palliative care services in regions with the least access.
\end{abstract}

J Natl Compr Canc Netw 2016;14(4):439-445

\section{Background}

Palliative care is specialized medical care for people with serious illness at any stage that focuses on optimizing patient quality of life. ${ }^{1}$ A multidisciplinary palliative care team will often include physicians, nurses, social workers, pharmacists, and spiritual counselors who collaborate with other medical providers. The palliative care team specializes in whole person assessment, symptom management, and advanced-care planning communication. In oncology, palliative care has increasing relevance given recent studies that link palliative care to improved patient quality of life, reduced cost of care, and even improved survival in patients with metastatic cancer. $^{2-9}$ In fact, current national clinical guidelines recommend early combined integration of palliative care with standard oncology care for any patient with metastatic cancer or high symptom burden. ${ }^{10}$

From ${ }^{a}$ Doris A. Howell Palliative Care Service, and the bepartment of Radiation Medicine and Applied Science, Moores Cancer Center, University of California San Diego, La Jolla, California.

Submitted February 5, 2016; accepted for publication March 1, 2016.

The authors have disclosed that they have no financial interests,

arrangements, affiliations, or commercial interests with the manufacturers
Survey studies have found an increase in the availability of palliative care across the United States, ${ }^{1}$ with a near 3-fold increase in the number of hospitals with palliative care teams over the past decade. Similarly, research involving single institutions or single health care networks has found an increase in the use of palliative care services. ${ }^{11-13}$ Despite the apparent increasing use of palliative care in select health care settings, we lack an understanding of how palliative care is delivered among patients with cancer across the nation as a whole. The purpose of this study was to characterize the evolution of palliative care services in oncology from a population-based standpoint. Specifically, we focused on defining the growth, use, and regional differences of palliative care delivery among elderly patients (aged $>65$ years) with advanced cancer.

of any products discussed in this article or their competitors. This study was supported by NIH KL2 RR031978 (J.D.M.) and NIH T32 (I.J.B.).

Correspondence: James D. Murphy, MD, MS, University of California, San

Diego, Department of Radiation Medicine and Applied Sciences, 3960

Health Sciences Drive, MC0865, La Jolla, CA 92093-0865.

E-mail: j2murphy@ucsd.edu 


\section{Methods}

\section{Data}

This study identified patients with cancer from the SEER-Medicare linked database. SEER represents a collection of individual cancer registries geographically spread across the United States accounting for approximately $28 \%$ of the US population. SEER collects information on incident cancer diagnoses within each cancer registry, including demographic, clinical, tumor-specific, and treatment-related information. Medicare provides federally funded health insurance for patients older than 65 years. The SEER-Medicare linked dataset combines patient information from SEER with claims information from the Medicare program. The Medicare claims data allows the ability to track longitudinal patterns of care and health outcomes beyond what SEER captures. Although some minor differences exist in elderly patients within SEER-Medicare compared with the greater United States, overall this unique population-based dataset allows researchers to follow patterns of care and outcomes from prior to diagnosis through the end of life. ${ }^{14}$ The Institutional Review Board of the University of California San Diego deemed this study exempt from review.

\section{Patients}

This study included patients 66 years of age and older with breast, prostate, lung, or colorectal cancer, which represent the 4 most common cancers in the United States. ${ }^{15}$ SEER-Medicare includes patients 65 years of age and older; however, we included patients 66 years of age and older to allow for a 1-year time window before diagnosis to ascertain preexisting comorbidity (described further later). From the SEER-Medicare database, we initially identified 166,124 patients with histologically confirmed distant metastatic cancer diagnosed between 2000 and 2009. We excluded patients with multiple primary tumors $(n=21,550)$, and those diagnosed at death or on autopsy $(n=299)$. This study focused on palliative care in the oncology setting; therefore, we excluded patients who died of noncancer causes $(n=12,043)$ and patients alive at the end of the follow-up period $(n=9,942)$ on December 31, 2010. As is standard in research involving SEER-Medicare, we excluded those with incomplete Medicare claims data. This resulted in excluding subjects without continuous Medicare Parts $A$ and $B$, or enrollment in Part $\mathrm{C}$ any time from 1 year before di- agnosis (to determine comorbidity) through death (to identify palliative care). The final study population included 83,022 subjects.

\section{Identifying Palliative Care Consultation}

Consultation with a palliative care provider was identified using the International Classification of Diseases, Ninth Revision (ICD-9) code V66.7 ("Encounter for Palliative Care") which is a V-code modifier for palliative care created in $1996 .{ }^{16}$ We identified inpatient and outpatient palliative care consultation separately by their appearance in their respective Medicare files. Note that in Medicare, enrollment into hospice is recorded separately from an encounter with palliative care. Hospice use has been characterized extensively elsewhere, and was not included in this analysis.

\section{Patient Covariates}

Patient-related demographic variables obtained in SEER included age at diagnosis, sex, race, marital status, and median household income. Median household income was estimated from the 2000 US Census using census tract data preferentially over zip code data, and divided into quartiles for analysis. Patients with missing household income $(n=22)$ were included in the bottom quartile. ${ }^{17}$ Comorbidity before cancer diagnosis was estimated using the Deyo adaptation of the Charlson comorbidity index, which uses inpatient and outpatient Medicare claims data the year prior to a patient's cancer diagnosis. ${ }^{18,19}$ Individual SEER cancer registries were reclassified into East (Connecticut and New Jersey), Midwest (Detroit and Iowa), South (Georgia, Kentucky, and Louisiana), and West (California, Hawaii, New Mexico, Seattle, and Utah). Care in a teaching hospital was defined as any indirect medical education payment during a hospitalization after cancer diagnosis.

\section{Regional Covariates}

This study evaluated the association between the regional use of cancer-related palliative care and regional health care characteristics. Regional geography was evaluated at the level of hospital referral regions. There are 306 hospital referral regions spread across the United States, and each distinct geographic region represents a predefined health care market for tertiary care services. ${ }^{20}$ We looked for correlations in the regional use of palliative care and various aggregate regional health care characteristics. Regional hospital and provider character- 
Regional Variation in Palliative Care

istics, obtained from the Dartmouth Atlas of Health Care, ${ }^{21}$ included the following variables: number of acute care hospital beds, primary care physicians, hematologist-oncologists, and total physicians. Regional health care use was evaluated with a measure of the average number of days spent in an inpatient hospital and intensive care unit (ICU) in the last 6 months of life, and the percentage of patients who die in a hospital compared with a nonhospital setting. Regional health care expenditure was expressed as the regional annual Medicare reimbursement per enrollee in that specific hospital referral region adjusted for race, age, sex, and geography. ${ }^{20}$

\section{Statistical Analysis}

Patient characteristics were stratified by presence or absence of the palliative care consultation ICD-9 code. Multivariable logistic regression models were used to determine the association between palliative care consult and specific patient characteristics. All patient covariates analyzed in the multivariable model were chosen a priori, and included patient demographic and clinical characteristics thought to potentially impact the use of palliative care services (covariates listed in Table 1). The ecologic analysis to determine associations between palliative care consultation and regional health care characteristics used an average rate of palliative care consultation per each hospital referral region. This average rate of palliative care consultation was further adjusted to account for underlying differences in patient age, race, and sex by adjustment to the 2000 US population. ${ }^{22}$ We grouped patients into quartiles based on the adjusted rates of palliative care use within each hospital referral region. We evaluated trends between quartiles of palliative care use and regional characteristics with a Cuzick's ${ }^{23}$ trend test. All statistical tests were 2 -sided, and $P$ values less than 0.05 were considered significant. Statistical analyses were performed with SAS version 9.4 (SAS Institute, Inc., Cary, NC).

\section{Results}

Among the 83,022 patients with cancer in this study, $5,042(6.1 \%)$ had a record of a palliative care consultation. The fraction of patients receiving a palliative care consultation increased steadily from 3.0\% in 2000 to $12.9 \%$ in 2009 (Figure 1). Most palliative care consultations occurred in the inpatient setting.
There were 4,186 patients $(83 \%)$ with only an inpatient consult, 599 (12\%) with only an outpatient consult, and 257 (5\%) with both an inpatient and outpatient consult. Additionally, a substantial portion of patients had palliative care consultations near the end of life. Among patients with a palliative care consultation, $77 \%$ had a consultation within a month of death and $37 \%$ had it in the last week of life (Figure 2). Less than $10 \%$ of patients had a palliative care consultation more than 3 months before death.

Table 1 demonstrates associations between patient-level characteristics and palliative care consultation. On multivariable analysis, older patients and male patients were less likely to undergo consultation from a palliative care provider. Among a small percentage of the identified cases, prostate cancer was more likely to have a palliative care consultation in contrast to other cancer types. Patients with higher preexisting comorbidity were less likely to receive palliative care consultation. The odds of a palliative care consultation nearly doubled among patients receiving care in a teaching hospital. Patients in the South had lower rates of palliative care use.

Analysis of data by hospital referral region revealed substantial geographic variability in the use of palliative care (Figure 3). The use of palliative care ranged from $0 \%$ to $14.1 \%$ from the lowest to the highest hospital referral region. Of note, $94 \%$ of hospital referral regions had an increase in the use of palliative care over the study period.

Associations between regional health care characteristics and palliative care are presented in Table 2. The likelihood of a palliative care consultation increased with decreased numbers of regional acute care hospital beds. The increased use of palliative care resources was associated with increased numbers of regional physicians per capita. Of note, no marker of aggressive care at the end of life on the regional level was associated with palliative care use. This included the regional average rates of hospitalization use or ICU use in the last 6 months of life, as well as percent of patients dying within a hospital setting. Finally, lower regional annual reimbursement per Medicare enrollee was associated with increased use of palliative care. Between the first and fourth quartile of palliative care use, the regional annual reimbursement decreased by $\$ 1,387$ per Medicare enrollee. 
Table 1. Patient-Level Predictors of Palliative Care Consultation

\begin{tabular}{|c|c|c|c|c|}
\hline \multirow[b]{2}{*}{ Characteristic } & \multicolumn{2}{|c|}{ Palliative Care Consult } & \multicolumn{2}{|c|}{ Multivariable Analysis } \\
\hline & Yes & No & $\begin{array}{l}\text { Odds Ratio } \\
(95 \% \mathrm{Cl})\end{array}$ & $P$ Value \\
\hline All patients & $5,042(6.1)$ & $77,980(93.9)$ & & \\
\hline \multicolumn{5}{|l|}{ Age, y } \\
\hline $66-69$ & $1,066(6.5)$ & $15,396(93.5)$ & 1 & - \\
\hline $70-74$ & $1,297(6.0)$ & $20,287(94.0)$ & $0.94(0.86-1.02)$ & .12 \\
\hline $75-79$ & $1,243(6.0)$ & $19,457(94.0)$ & $0.92(0.84-1.00)$ & .05 \\
\hline $80-84$ & $880(6.0)$ & $13,911(94.1)$ & $0.87(0.79-0.95)$ & .003 \\
\hline$\geq 85$ & $556(5.9)$ & $8,929(94.1)$ & $0.79(0.71-0.89)$ & $<.0001$ \\
\hline \multicolumn{5}{|l|}{ Sex } \\
\hline Female & $2,598(6.5)$ & $37,289(93.5)$ & 1 & - \\
\hline Male & $2,444(5.7)$ & 40,691 (94.3) & $0.85(0.80-0.91)$ & $<.0001$ \\
\hline \multicolumn{5}{|l|}{ Race } \\
\hline White & $4,205(5.9)$ & $66,959(94.1)$ & 1 & - \\
\hline Black & $500(6.1)$ & $7,657(93.9)$ & $1.10(0.99-1.21)$ & .07 \\
\hline Asian or Pacific Islander & $307(9.1)$ & $3,056(90.9)$ & $1.11(0.97-1.26)$ & .13 \\
\hline Other/Unknown & $30(8.9)$ & 308 (91.1) & $1.17(0.79-1.71)$ & .43 \\
\hline \multicolumn{5}{|l|}{ Ethnicity } \\
\hline Hispanic & $253(7.1)$ & $3,292(92.9)$ & $0.93(0.81-1.07)$ & .32 \\
\hline Non-Hispanic & $4,789(6.0)$ & $74,688(94.0)$ & 1 & - \\
\hline \multicolumn{5}{|l|}{ Marital status } \\
\hline Married & $2,477(6.1)$ & $38,226(93.9)$ & $1.05(0.99-1.12)$ & .10 \\
\hline Non-married & $2,565(6.1)$ & $39,754(93.9)$ & 1 & - \\
\hline \multicolumn{5}{|l|}{ Charlson score } \\
\hline 0 & $2,431(5.6)$ & 40,793 (94.4) & 1 & - \\
\hline 1 & $1,424(6.6)$ & $20,088(93.4)$ & $1.03(0.96-1.11)$ & .38 \\
\hline 2 & $647(6.6)$ & $9,149(93.4)$ & $1.02(0.93-1.11)$ & .75 \\
\hline$\geq 3$ & $540(6.4)$ & $7,950(93.6)$ & $0.90(0.82-1.00)$ & .04 \\
\hline \multicolumn{5}{|l|}{ Tumor site } \\
\hline Breast & $258(6.1)$ & $3,958(93.9)$ & $0.99(0.87-1.14)$ & .93 \\
\hline Colorectal & $812(5.6)$ & $13,721(94.4)$ & $0.94(0.87-1.02)$ & .12 \\
\hline Lung & $3,731(6.2)$ & $56,669(93.8)$ & 1 & - \\
\hline Prostate & $241(6.2)$ & $3,632(93.8)$ & $1.21(1.05-1.40)$ & .01 \\
\hline \multicolumn{5}{|l|}{ Year of diagnosis } \\
\hline 2000 & $245(3.0)$ & $7,865(97.0)$ & 1 & - \\
\hline 2001 & $247(3.0)$ & $8,063(97.0)$ & $0.97(0.81-1.16)$ & .76 \\
\hline 2002 & $292(3.4)$ & $8,206(96.6)$ & $1.14(0.96-1.36)$ & .13 \\
\hline 2003 & $358(4.0)$ & $8,616(96.0)$ & $1.31(1.11-1.54)$ & .002 \\
\hline 2004 & $442(4.8)$ & $8,803(95.2)$ & $1.59(1.35-1.86)$ & $<.0001$ \\
\hline 2005 & $487(5.4)$ & $8,572(94.6)$ & $1.80(1.54-2.11)$ & $<.0001$ \\
\hline 2006 & $576(6.6)$ & $8,111(93.4)$ & $2.25(1.93-2.62)$ & $<.0001$ \\
\hline 2007 & $732(9.0)$ & $7,387(91.0)$ & $3.13(2.70-3.63)$ & $<.0001$ \\
\hline 2008 & $810(10.9)$ & $6,602(89.1)$ & $3.94(3.39-4.56)$ & $<.0001$ \\
\hline 2009 & $853(12.9)$ & $5,755(87.1)$ & $4.83(4.17-5.60)$ & $<.0001$ \\
\hline \multicolumn{5}{|l|}{ Geographic area } \\
\hline East & $1,148(6.7)$ & $15,971(93.3)$ & 1 & - \\
\hline Midwest & $827(6.9)$ & $11,231(93.1)$ & $0.94(0.85-1.03)$ & .19 \\
\hline South & $728(3.2)$ & $22,228(96.8)$ & $0.53(0.48-0.58)$ & $<.0001$ \\
\hline West & $2,339(7.6)$ & $28,550(92.4)$ & 1 & - \\
\hline \multicolumn{5}{|l|}{ Population density } \\
\hline Urban & $4,328(6.4)$ & $63,642(93.6)$ & $0.99(0.91-1.08)$ & .78 \\
\hline Rural & $714(4.7)$ & $14,338(95.3)$ & 1 & - \\
\hline \multicolumn{5}{|l|}{ Teaching hospital } \\
\hline Yes & $3,128(8.3)$ & $34,778(91.8)$ & $1.97(1.85-2.10)$ & $<.0001$ \\
\hline No & $1,914(4.2)$ & $43,202(95.8)$ & 1 & - \\
\hline
\end{tabular}


Regional Variation in Palliative Care

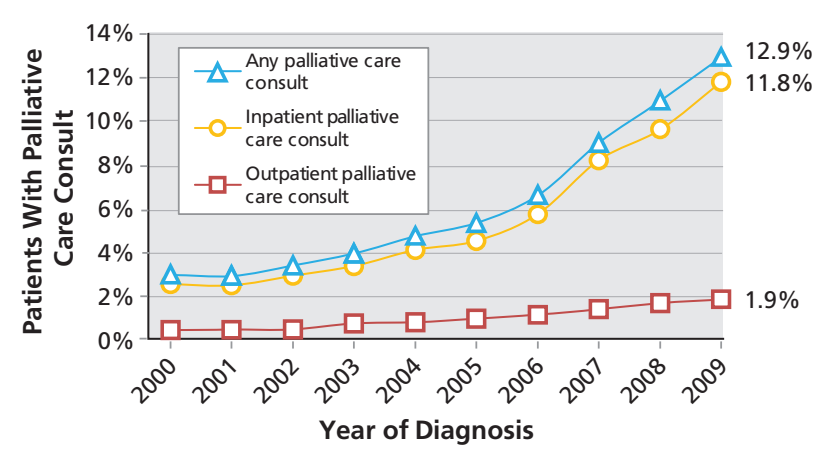

Figure 1. Rates of palliative care over study period.

\section{Discussion}

This current study found a steady increase in the rate of palliative care consultation across the United States over the past decade. Our findings describing the use of palliative care services parallel and further validate other research describing the availability of palliative care. Morrison ${ }^{24}$ described palliative care penetration highest in the New England (88\% of hospitals), Pacific (77\% of hospitals), and mid-Atlantic (77\% of hospitals) states and lowest in the west south central (43\% of hospitals) and east south central ( $42 \%$ of hospitals) states. Over the past decade, the number of hospitals with palliative care teams has nearly tripled. In 2012, a total of 1,734 of 2,844 hospitals with 50 beds or more reported having a palliative care team, and these teams served an estimated 6 million patients. ${ }^{1}$ In 2006, midway through our study period, the American Board of Medical Specialties and the Accreditation Council for Graduate Medical Education recognized Hospice and Palliative Medicine as a subspecialty with its own board examination. We found a steady increase in the use of palliative care before and after 2006.

A notable finding of our study highlights the observation that palliative care interventions occur very near the end of life, most often in an inpatient setting. In fact, more than one-third of patients receiving palliative care did so in the last week of life.

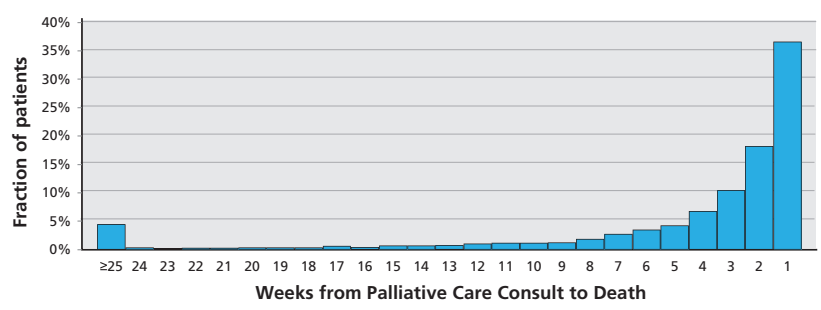

Figure 2. Timing of palliative care consultation.
These findings echo survey data from US cancer centers showing that palliative care referrals occurred late in patients' disease trajectories. ${ }^{11}$ Additional survey data from Canada also found late referrals to palliative care, often in response to patients with uncontrolled symptoms. ${ }^{25}$ The landmark study by Temel et al, ${ }^{5}$ published in 2010, demonstrated the impact of early palliative intervention. This study found that patients with metastatic lung cancer had improved quality of life and survival when early palliative care was integrated with standard oncology care. ASCO and NCCN both have clinical guidelines that recommend early integration of palliative care among patients with incurable cancer. ${ }^{10,26}$ It is important to consider that these guidelines were published after our study period (2000-2009), although we found that most palliative care is delivered in the inpatient setting near the end of life. This suggests that palliative care today is far from achieving the recommended early, simultaneous integration delivery model into standard oncology care.

Beyond the timing of palliative care, our study found considerable regional differences in the use of palliative care across the United States. Our analysis by hospital referral region revealed that the rates of palliative care among patients with metastatic cancer ranged from a low of $0 \%$ to a high of $14 \%$. We did find that the fraction of patients receiving palliative care consultation increased in regions with more physicians per capita and in regions with lower Medicare expenditure per enrollee. Importantly, the association between palliative care and regional Medicare expenditure in this study does not necessarily imply causality. For example, we cannot assume that the increased use of palliative care led to decreased health care expenditure. This relationship may simply indicate that underlying patient or regional factors led to both increased use of palliative care and decreased use of health care resources. Of note, decreased health care cost is a byproduct not a goal of palliative care consultation. Despite the lack of causality, a recent study found decreased health care cost among hospice enrollees compared with control patients. ${ }^{27}$ Additional research on administrative data from 8 hospitals found that patients undergoing palliative care had substantially decreased health care costs associated with hospital admissions. ${ }^{12}$ Future prospective research or carefully controlled retrospective cohort studies are needed to 


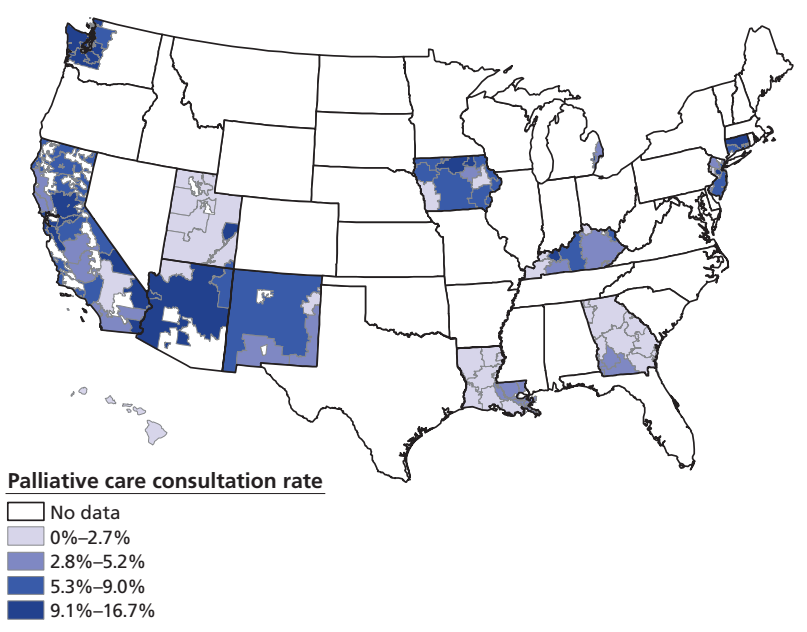

Figure 3. Geographic variation of palliative care consultation.

fully understand the health economic implications of palliative care.

This population-based study has limitations worth considering. Given the nature of SEER-Medicare data, we cannot readily validate the sensitivity or specificity of the ICD-9 code for palliative care. Although the code appears specific to palliative care consultation, undercoding is likely, which could lead us to underestimate palliative care delivery in el- derly patients with cancer. Additionally, our analysis did not include patients younger than 66 years. We found increasing rates of palliative care among our youngest cohort of patients, which could indicate higher rates among patients younger than 65 years; however, we do not know for certain if these results generalize to a younger population. This representative sample is also limited to patients with cancer located in SEER locations, and therefore our findings my not translate to regions outside of SEER. Finally, we restricted our analysis to breast, colorectal, lung, and prostate cancers, and excluded patients in managed care organizations; therefore, our results may not generalize to other cancers, patients without cancer, or different health care networks.

Despite these limitations, in this population of Medicare patients with advanced cancer we found that the rates of palliative care have steadily increased over the past decade, although consultations appear to occur very near the end of life and mostly in the inpatient setting. Additionally, geographic location substantially influences whether a patient will receive palliative care. These findings provide useful information on potential barriers in palliative care dissemination and provide guidance for future areas of palliative care growth.

\section{Table 2. Regional Characteristics of Palliative Care Consultation}

\begin{tabular}{|c|c|c|c|c|c|}
\hline \multirow[b]{2}{*}{ Regional Characteristic } & \multicolumn{4}{|c|}{ Palliative Care Consultation Rate by Hospital Referral Region ${ }^{a}$} & \multirow[b]{2}{*}{$\begin{array}{l}P \text { Value } \\
\text { for Trend }\end{array}$} \\
\hline & $\begin{array}{l}\text { 1st Quartile } \\
(0.0 \%-3.5 \%)\end{array}$ & $\begin{array}{l}\text { 2nd Quartile } \\
(3.6 \%-5.7 \%)\end{array}$ & $\begin{array}{l}\text { 3rd Quartile } \\
(5.7 \%-7.9 \%)\end{array}$ & $\begin{array}{l}\text { 4th Quartile } \\
(7.9 \%-14.1 \%)\end{array}$ & \\
\hline Number of hospital referral regions & 32 & 24 & 12 & 25 & \\
\hline Number of patients & 21,129 & 24,422 & 18,083 & 19,388 & \\
\hline \multicolumn{6}{|l|}{ Regional characteristic } \\
\hline $\begin{array}{l}\text { Acute care hospital beds (per 1,000 } \\
\text { residents) }\end{array}$ & 2.89 & 2.39 & 2.38 & 2.09 & $<.0001$ \\
\hline Total physicians (per 100,000 residents) & 178 & 199 & 198 & 214 & .0001 \\
\hline $\begin{array}{l}\text { Primary care physicians (per 100,000 } \\
\text { residents) }\end{array}$ & 64 & 71 & 73 & 79 & $<.0001$ \\
\hline $\begin{array}{l}\text { Hematologist-oncologists (per 100,000 } \\
\text { residents) }\end{array}$ & 2.44 & 2.75 & 3.03 & 3.08 & .03 \\
\hline $\begin{array}{l}\text { Hospitalization days in last } 6 \text { months of } \\
\text { life }\end{array}$ & 9.4 & 10.2 & 9.4 & 8.9 & .11 \\
\hline ICU days in last 6 months of life & 3.3 & 3.56 & 3.06 & 3.23 & .57 \\
\hline Percent of patients dying in a hospital & 27 & 28 & 27 & 26 & .33 \\
\hline $\begin{array}{l}\text { Regional Medicare reimbursement per } \\
\text { enrollee }\end{array}$ & $\$ 8,496$ & $\$ 7,856$ & $\$ 7,225$ & $\$ 7,109$ & $<.0001$ \\
\hline
\end{tabular}

Abbreviation: ICU, intensive care unit.

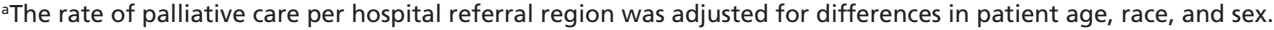

${ }^{\mathrm{b}} P$ value represents Cuzick's trend test. 


\section{Acknowledgments}

This study used the linked SEER-Medicare database. The interpretation and reporting of these data are the sole responsibility of the authors. The authors acknowledge the efforts of the Applied Research Program, NCI; the Office of Research, Development and Information, Centers for Medicare \& Medicaid Services; Information Management Services, Inc; and the SEER Program tumor registries in the creation of the SEER-Medicare database.

\section{References}

1. Center for Medicare \& Medicaid Services. Medicare Benefit Policy Manual. Chapter 9: Coverage of Hospital Services Under Hospital Insurance. Available at: http://www.cms.gov/Regulations-and-Guidance/ Guidance/Manuals/downloads/bp102c09.pdf. Accessed 17 July 2014.

2. Rabow MW, Dibble SL, Pantilat SZ, et al. The comprehensive care team: a controlled trial of outpatient palliative medicine consultation. Arch Intern Med 2004;164:83-91.

3. Smith TJ, Hillner BE. Bending the cost curve in cancer care. N Engl J Med 2011;364:2060-2065.

4. Bakitas M, Lyons KD, Hegel MT, et al. Effects of a palliative care intervention on clinical outcomes in patients with advanced cancer: the project ENABLE II randomized controlled trial. JAMA 2009;302:741-749.

5. Temel JS, Greer JA, Muzikansky A, et al. Early palliative care for patients with metastatic non-small-cell lung cancer. N Engl J Med 2010;363:733742 .

6. Yoong J, Park ER, Greer JA, et al. Early palliative care in advanced lung cancer: a qualitative study. JAMA Intern Med 2013;173:283-290.

7. Zimmermann C, Swami N, Krzyzanowska M, et al. Early palliative care for patients with advanced cancer: a cluster-randomised controlled trial. Lancet 2014;383:1721-1730.

8. Bakitas MA, Tosteson TD, Li Z, et al. Early versus delayed initiation of concurrent palliative oncology care: Patient outcomes in the ENABLE III randomized controlled trial. J Clin Oncol 2015;33:1438-1445.

9. Ferrell B, Sun V, Hurria A, et al. Interdisciplinary palliative care for patients with lung cancer. J Pain Symptom Manage 2015;50:758-767.
10. Smith TJ, Temin S, Alesi ER, et al. American Society of Clinical Oncology provisional clinical opinion: the integration of palliative care into standard oncology care. J Clin Oncol 2012;30:880-887.

11. Hui D, Elsayem A, De la Cruz M, et al. Availability and integration of palliative care at US cancer centers. JAMA 2010;303:1054-1061.

12. Morrison RS, Augustin R, Souvanna P, et al. America's care of serious illness: a state-by-state report card on access to palliative care in our nation's hospitals. J Palliat Med 2011;14:1094-1096.

13. Hughes MT, Smith TJ. The growth of palliative care in the United States. Annu Rev Public Health 2014;35:459-475.

14. Warren JL, Klabunde CN, Schrag D, et al. Overview of the SEERMedicare data: content, research applications, and generalizability to the United States elderly population. Med Care 2002;40(8 Suppl):IV-3-IV-18.

15. Siegel RL, Miller KD, Jemal A. Cancer statistics, 2015. CA Cancer J Clin 2015;65:5-29.

16. Capello CF, Meier DE, Cassel CK. Payment code for hospital-based palliative care: help or hindrance? J Palliat Med 1998;1:155-163.

17. Krieger N. Overcoming the absence of socioeconomic data in medical records: validation and application of a census-based methodology. Am J Public Health 199282:703-710.

18. Charlson ME, Pompei $P$, Ales KL, et al. A new method of classifying prognostic comorbidity in longitudinal studies: development and validation. J Chronic Dis 1987;40:373-383.

19. Deyo RA, Cherkin DC, Ciol MA. Adapting a clinical comorbidity index for use with ICD-9-CM administrative databases. J Clin Epidemiol 1992;45:613-619.

20. Wennberg JE, McAndrew Cooper M, eds. The Dartmouth Atlas of Health Care. Health Forum, Inc.: Chicago, IL;1999.

21. O'Gorman P, McMillan DC, McArdle CS. Prognostic factors in advanced gastrointestinal cancer patients with weight loss. Nutr Cancer 2000;37:3640.

22. Falconer JS, Fearon KC, Ross JA, et al. Acute-phase protein response and survival duration of patients with pancreatic cancer. Cancer 1995;75:2077-2082.

23. Cuzick J. A Wilcoxon-type test for trend. Stat Med 1985;4:543-547.

24. Morrison SR, Meier DE. America's care of serious illness: 2015 state-bystate report card on access to palliative care in our Nation's Hospitals. Available at: https://registry.capc.org/wp-content/uploads/2016/01/2015. State-by-state-Report-Card.pdf. Accessed March 21, 2016.

25. Wentlandt K, Krzyzanowska MK, Swami N, et al. Referral practices of oncologists to specialized palliative care. J Clin Oncol 2012;30:4380-4386.

26. Fearon KC. Cancer cachexia: developing multimodal therapy for a multidimensional problem. Eur J Cancer 2008;44:1124-1132.

27. Obermeyer Z, Makar M, Abujaber S, et al. Association between the Medicare hospice benefit and health care utilization and costs for patients with poor-prognosis cancer. JAMA 2014;312:1888-1896. 